\title{
Mercado farmacéutico en México: tamaño, valor y concentración
}

\author{
Sandra Torres Guerra ${ }^{1}$ y Juan Pablo Gutiérrez ${ }^{1,2}$
}

Forma de citar Torres Guerra S, Gutiérrez JP. Mercado farmacéutico en México: tamaño, valor y concentración. Rev Panam Salud Publica. 2009;26(1):46-50.

RESUMEN Objetivo. Caracterizar el mercado de medicamentos de México en cuanto a su tamaño, organización, poder de mercado de las empresas y capacidad de negociación de los consumidores. Métodos. Estudio descriptivo a partir de los datos de los Censos Económicos de 2004 y los informes de Intercontinental Marketing Services. Se obtuvieron los montos y volúmenes de ventas de las empresas del sector farmacéutico mexicano de 2002 a 2005 y se calcularon el indice de Herfindahl-Hirschman (IHH) y su inverso como indicadores del grado de concentración del mercado, así como la elasticidad del precio por un producto índice.

Resultados. El valor total de los productos elaborados por el sector farmacéutico fue de 115 mil millones en pesos de 2006, de los que 99,0\% correspondió a las empresas clasificadas como grandes. Este monto representó 1,2\% del producto interno bruto nacional de ese año $(20,0 \%$ de la participación del sector salud, estimada en 6,0\%) y 3,9\% del valor de los productos de manufactura. El IHH del mercado farmacéutico mexicano en el período analizado fue de alrededor de 0,04, aunque con una reducción sostenida, y su inverso se redujo de 23 a 26. La elasticidad del precio de los productos farmacéuticos entre 2003 y 2005 fue mínima $(0,007,0,003$ $y-0,002)$.

Conclusiones. Este trabajo constituye una primera caracterización del mercado farmacéutico mexicano, uno de los sectores más dinámicos de la economía nacional. Se comprobó que se trata de un mercado oligopólico e inelástico, por lo que se justifica la creación de mecanismos regulatorios más sólidos que reduzcan el poder de los productores en favor de los consumidores.

Palabras clave Comercialización de medicamentos, medicamentos de prescripción, medicamentos sin prescripción, México.

La industria farmacéutica es uno de los sectores más dinámicos e importantes de la economía mundial en términos de recursos movilizados. Si bien se trata de uno de los sectores económicos en que la globalización es más evidente (los productos se desarrollan para un mer-

1 Centro de Investigación en Evaluación y Encuestas, Instituto Nacional de Salud Pública, Cuernavaca, México. La correspondencia se debe dirigir a Juan Pablo Gutiérrez, Centro de Investigación en Evaluación y Encuestas, Instituto Nacional de Salud Pública, Av. Universidad No. 655, Sta. María Ahuacatitlán, Cuernavaca, Morelos 62508, México. Correo electrónico: jpgutier@insp.mx

2 Unidad de Política de Salud, Escuela Londinense de Higiene y Medicina Tropical, Londres, Reino Unido. cado global), las particularidades de cada país hacen que los mercados nacionales tengan sus propias dinámicas $\mathrm{y}$ características.

El mercado farmacéutico mexicano es uno de los de mayor dinamismo en América Latina debido al crecimiento que ha registrado y se ubica como el noveno en importancia en el mundo $(1,2)$. Según algunas estimaciones, el sector farmacéutico crecerá en los mercados emergentes, entre los que se incluye México, entre $14 \%$ y $15 \%$ (3).

Las reformas relacionadas con la provisión de servicios de salud que se llevan a cabo en México —en particular las re- formas a la Ley del Instituto Mexicano del Seguro Social y a la Ley de Salud que han dado lugar a la formalización del sistema de protección en salud, el Seguro Popular - han llevado a un notable incremento en el monto de los recursos públicos destinados a la compra de medicamentos. Se espera que, al mismo tiempo, esto permita reducir la compra directa de los mismos por parte del consumidor final (4).

De acuerdo con la teoría económica, hay algunos bienes cuya demanda es muy sensible al precio, de manera que las variaciones en el precio de venta al público provocan cambios proporcionales 
en la demanda (bienes elásticos respecto al precio); mientras en otros bienes, las variaciones en el precio ocasionan cambios menos proporcionales $-\mathrm{O}$ incluso nulos- en la demanda (bienes inelásticos). Como la capacidad de decisión sobre el consumo es reducida en el caso de los medicamentos, estos se consideran bienes inelásticos respecto al precio.

Mientras menos elasticidad tiene un producto, mayor poder tienen los productores para determinar los precios en detrimento del consumidor. La situación más alejada del ideal es la de un mercado monopólico, en el que la demanda resulta totalmente inelástica con relación al precio. En este caso se hace indispensable establecer regulaciones y estimular la competencia entre varias empresas para evitar precios excesivos. Es preferible un sector más cercano a la competencia perfecta que un mercado concentrado.

El análisis del grado de concentración del mercado - para determinar la proporción que corresponde a cada una de las empresas dentro del mercado- permitirá caracterizar el mercado y conocer su grado de aproximación a un estado de competencia perfecta.

La caracterización de la segmentación entre el mercado de medicamentos de prescripción o controlado (también llamado ético por la industria) y el de medicamentos que no requieren prescripción o libre (también llamado popular) permite identificar los distintos nichos y estrategias de mercado y de la industria. Se debe tener en cuenta, no obstante, que el comportamiento de esos dos segmentos responde a factores diferentes, ya que las regulaciones pueden tener un efecto mayor sobre el mercado controlado que sobre el libre.

El concepto de elasticidad del precio, dada por la variación porcentual de la demanda de un producto - en este caso farmacéutico- en función de las variaciones porcentuales en el precio, refleja la relación de poder, en términos de determinación de precios, entre los productores y los consumidores (5). Este indicador refleja la sensibilidad de la demanda ante cambios en el precio: a mayor elasticidad del precio de un producto más se puede afectar la demanda, por lo que los productores deben tener más cuidado al incrementar los precios (5).

El objetivo del presente trabajo es caracterizar el mercado de medicamentos de México en cuanto a su tamaño, organización, poder de mercado de las em- presas y capacidad de negociación de los consumidores.

\section{MATERIALES Y MÉTODOS}

Se realizó un estudio descriptivo a partir de las estadísticas económicas nacionales de carácter público, como los Censos Económicos de 2004 y los informes de Intercontinental Marketing Services, en particular de su rama de salud (IMS Health).

Para describir el mercado mexicano de productos farmacéuticos según su peso relativo en la economía nacional - la contribución porcentual del sector al producto interno bruto- y caracterizarlo según el número y el tamaño de las empresas se analizó el valor total de los productos elaborados en las clases "fabricación de materias primas para la industria farmacéutica" (código 325411 en el Sistema de Clasificación Industrial de América del Norte) y "fabricación de preparaciones farmacéuticas" (código 325412) (6). Los datos disponibles de los Censos Económicos de 2004 abarcan los establecimientos que brindaron información detallada - ya se dediquen a manufacturar materia prima o producto final-, independientemente de si el destino de su producción es para uso humano o veterinario.

Para hacer un análisis más específico del sector dedicado a productos finales de uso humano, se utilizaron los datos de IMS Health sobre las ventas anuales de las empresas del sector farmacéutico mexicano, tanto en valor como en unidades de medicamentos, en el período de 2002 a 2005. Se tomó en cuenta la segmentación tanto del mercado controlado como del libre. Los datos de las ventas anuales se homogeneizaron mediante el índice de inflación calculado por el Banco de México para el sector farmacéutico, según el valor del peso mexicano en junio de 2006 (7).

Con esta información se calculó el valor total del mercado - como la suma de las ventas de todas las empresas (en pesos mexicanos) en cada segmento-, el porcentaje del mercado que le correspondió a cada empresa por año - como el cociente del valor de las ventas de la empresa sobre el valor total de las ventas- y los porcentajes de los segmentos controlado y libre.

Se calculó el índice de HerfindahlHirschman (IHH) para cada año, como medida del grado de concentración del mercado, mediante la siguiente fórmula:

$$
\mathrm{IHH}=\sum\left(s_{i}\right)^{2}
$$

donde, $s$ es el total de las ventas de la empresa $i$ entre el total de las ventas del sector en un año dado. El valor de IHH tiende a cero cuando el número de empresas en el mercado tiende al infinito con una participación similar, y es 1 cuando existe una sola empresa en el mercado (8).

Como una medida complementaria para caracterizar el mercado, se calculó el inverso del IHH, que indica el número de empresas de tamaño similar para un valor dado de IHH y con ello el nivel de competencia existente.

Para estimar la elasticidad del precio, se calculó el precio unitario de un producto índice a partir del cociente del volumen de sus ventas en unidades y el valor de las ventas en pesos mexicanos para cada una de las diferentes empresas (ajustado previamente por la inflación a pesos mexicanos de 2006). Aunque existe una amplia heterogeneidad de productos en el sector farmacéutico (desde antibióticos hasta vitaminas), el análisis basado en un producto índice facilita las comparaciones agregadas sin introducir sesgos que afecten la interpretación de los resultados.

Se calculó la elasticidad del precio (Eqp) entre cada par de años sucesivos entre 2002 y 2005 mediante la siguiente fórmula:

$$
\operatorname{Eqp}=(\Delta \% \mathrm{q} / \Delta \% \mathrm{p})(\mathrm{p} / \mathrm{q})
$$

donde, $\mathrm{p}$ es el precio; $\mathrm{q}$ es la cantidad de producto; y $\Delta \%$ es el cambio porcentual con respecto al año anterior.

Todos los cálculos se llevaron a cabo mediante el paquete estadístico Stata 8.2 (Stata Corporation, Texas, Estados Unidos de América).

\section{RESULTADOS}

De acuerdo con los datos de los Censos Económicos de 2004, en México había 480 empresas dedicadas a la producción de materias primas para la industria farmacéutica o de preparaciones farmacéuticas; de ellas, $236(49,2 \%)$ se clasificaron como establecimientos grandes por tener 100 empleados o más.

En conjunto, estas empresas tenían una plantilla laboral de cerca de $62000 \mathrm{em}$ pleados, de los cuales $54000(87,1 \%)$ trabajaban en las empresas grandes. El tamaño promedio de las pequeñas y medianas empresas (PYMES) fue de $33 \mathrm{em}$ - 
pleados, en tanto que el de las grandes fue de 229. En su conjunto, las empresas del sector farmacéutico representaban aproximadamente $0,2 \%$ del total de las empresas de manufactura en México y ocupaban a 1,5\% del personal empleado en este tipo de industria. De esta forma, la industria farmacéutica mostró una alta intensidad en el uso de recursos humanos (mayor porcentaje de personas que de empresas) y un valor agregado relativamente elevado (mayor porcentaje del valor de los productos que de los recursos humanos empleados).

El valor total de los productos elaborados por el sector fue de 94 mil millones de pesos (115 mil millones en pesos de 2006), de los que 99,0\% correspondió a las empresas clasificadas como grandes. Este monto representó $1,2 \%$ del producto interno bruto nacional de ese año $(20,0 \%$ de la participación del sector salud, estimada en 6,0\%) y $3,9 \%$ del valor de los productos de manufactura. Esto ubicaba a la industria farmacéutica en el tercer lugar en términos del valor de producción, solo por debajo de la refinación de petróleo y la fabricación de productos petroquímicos.

El valor total de las ventas de los productos de uso humano en los años analizados representó $82,8 \%$ del monto total de ventas de toda la industria farmacéutica, que en 2003 fue de 112000 millones de pesos mexicanos de 2006. Para los productos de uso humano, según el tipo de mercados, las ventas de medicamentos controlados en el período estudiado fueron 4,3 veces mayores que las de medicamentos libres, los cuales representaron en promedio 18,8\% del total del mercado para esos cuatro años (cuadro 1). Las ventas del sector mostraron crecimientos en todos los años, si bien se observaron grandes variaciones que iban de 1,3\% entre 2003 y 2004 hasta 6,0\% entre 2002 y 2003 (cuadro 1). En total, el mercado creció 11,8\% entre 2002 y 2005.

Según los datos de IMS Health para 299 empresas dedicadas a la manufactura y venta de productos farmacéuticos en México (62,3\% del total incluido en los Censos Económicos de 2004), 20 empresas concentraron entre 86,0 y $90,3 \%$ del mercado mexicano en los años analizados (cuadro 2). De esas empresas, las cinco primeras concentraron $32,1 \%$ del mercado en 2005, y a las 10 primeras correspondió $53,4 \%$, lo que confirma que se trata de un mercado altamente concentrado en un grupo reducido de empresas (oligopolio).

CUADRO 1. Comportamiento del mercado farmacéutico de México entre 2002 y 2005

\begin{tabular}{lccccccc}
\hline & \multicolumn{6}{c}{ Ventas totales $^{\mathrm{b}}$ y cambio porcentual anual $(\Delta \%)$} \\
\cline { 2 - 8 } $\begin{array}{c}\text { Tipo de } \\
\text { mercado }^{\mathrm{a}}\end{array}$ & 2002 & 2003 & $\begin{array}{c}\Delta \% \\
2003 / 2002\end{array}$ & 2004 & $\begin{array}{c}\Delta \% \\
2004 / 2003\end{array}$ & 2005 & $2005 / 2004$ \\
\hline Controlado & 70609 & 75409 & 6,8 & 76295 & 1,2 & 79770 & 4,6 \\
Libre & 16868 & 17295 & 2,5 & 17620 & 1,9 & 18027 & 2,3 \\
Total & 87477 & 92705 & 6,0 & 93916 & 1,3 & 97797 & 4,1 \\
\hline
\end{tabular}

a Por mercado controlado se entiende la venta de medicamentos de prescripción y por mercado libre la ventas de medicamentos que no requieren prescripción.

b Ventas en millones de pesos mexicanos de 2006

CUADRO 2. Segmento del mercado dominado por las principales empresas del sector farmacéutico de México entre 2002 y 2005

\begin{tabular}{|c|c|c|c|c|c|c|c|}
\hline \multirow[b]{2}{*}{ Empresa } & \multicolumn{7}{|c|}{ Segmento del mercado total (\%) y cambio porcentual anual $(\Delta \%)$} \\
\hline & 2002 & 2003 & $\begin{array}{c}\Delta \% \\
2003 / 2002\end{array}$ & 2004 & $\begin{array}{c}\Delta \% \\
2004 / 2003\end{array}$ & 2005 & $\begin{array}{c}\Delta \% \\
2005 / 2004\end{array}$ \\
\hline Pfizer & 8,6 & 8,4 & $-2,3$ & 8,4 & 0,0 & 8,0 & $-4,8$ \\
\hline Sanofi-Aventis & 8,0 & 7,7 & $-3,8$ & 7,5 & $-2,6$ & 7,5 & 0,0 \\
\hline Roche & 6,9 & 6,8 & $-1,4$ & 6,1 & $-10,3$ & 5,6 & $-8,2$ \\
\hline GlaxoSmithKline & 6,2 & 5,8 & $-6,5$ & 5,3 & $-8,6$ & 4,9 & $-7,5$ \\
\hline Schering Plough & 6,2 & 6,4 & 3,2 & 6,4 & 0,0 & 6,1 & $-4,7$ \\
\hline Novartis & 5,5 & 5,6 & 1,8 & 5,4 & $-3,6$ & 5,1 & $-5,6$ \\
\hline Boehringer Ingelheim & 4,6 & 4,4 & $-4,3$ & 4,3 & $-2,3$ & 4,3 & 0,0 \\
\hline Bayer & 4,3 & 4,3 & 0,0 & 4,4 & 2,3 & 4,4 & 0,0 \\
\hline Bristol Myer Squib & 4,3 & 4,2 & $-2,3$ & 3,6 & $-14,3$ & 3,4 & $-5,6$ \\
\hline Johnson and Johnson & 3,9 & 3,8 & $-2,6$ & 4,0 & 5,3 & 4,1 & 2,5 \\
\hline Home Products & 3,1 & 2,9 & $-6,5$ & 2,7 & $-6,9$ & 2,4 & $-11,1$ \\
\hline Merck Sharp \& Dohme & 3,1 & 3,1 & 0,0 & 3,0 & $-3,2$ & 2,7 & $-10,0$ \\
\hline Altana Pharma & 2,7 & 2,7 & 0,0 & 2,7 & 0,0 & 2,8 & 3,7 \\
\hline Senosiain & 2,5 & 2,5 & 0,0 & 2,7 & 8,0 & 2,6 & $-3,7$ \\
\hline Abbott & 2,2 & 2,1 & $-4,5$ & 2,1 & 0,0 & 2,1 & 0,0 \\
\hline AstraZeneca & 2,1 & 2,4 & 14,3 & 2,9 & 20,8 & 3,0 & 3,4 \\
\hline Valeant Pharmaceuticals Int. & 2,0 & 2,0 & 0,0 & 2,0 & 0,0 & 2,3 & 15,0 \\
\hline Merck & 1,9 & 2,1 & 10,5 & 2,5 & 19,0 & 2,7 & 8,0 \\
\hline Lilly & 1,8 & 1,8 & 0,0 & 1,9 & 5,6 & 2,2 & 15,8 \\
\hline Sanfer & 1,8 & 1,8 & 0,0 & 1,8 & 0,0 & 1,8 & 0,0 \\
\hline Otras & 18,3 & 19,2 & 4,9 & 20,3 & 5,7 & 22,0 & 8,4 \\
\hline
\end{tabular}

Se observaron, no obstante, ligeros cambios en cuanto al poder de mercado en los años analizados: mientras la concentración porcentual del mercado de la mayoría de las empresas que encabezan la lista sufrió reducciones, el poder de las empresas que ocupaban posiciones inferiores se incrementó (cuadro 2).

El valor del IHH se mantuvo relativamente estable durante los cuatro años analizados (alrededor de 0,04), aunque con una reducción sostenida que llegó a $11,6 \%$ entre 2002 y 2005, lo que podría indicar que el mercado tiende a una mayor competencia (cuadro 3). Esta tendencia se manifestó también en el inverso del IHH, cuyo valor se incrementó de 23 a 26 entre 2002 y 2005, es decir, aumentó el número de empresas con poder en el mercado.

Finalmente, la elasticidad del precio de los productos farmacéuticos fue mínima a lo largo del período (cuadro 3). Aunque se observó una tendencia a au-
CUADRO 3. Indice de Herfindahl-Hirschman (IHH), su inverso y elasticidad del precio en el mercado farmacéutico de México entre 20022005

\begin{tabular}{cccc}
\hline Año & $\mathrm{IHH}$ & $\begin{array}{c}\text { Inverso } \\
\text { de } I H H^{\mathrm{a}}\end{array}$ & $\begin{array}{c}\text { Elasticidad } \\
\text { del precio }\end{array}$ \\
\hline 2002 & 0,043 & 23 & NA \\
2003 & 0,042 & 24 & 0,007 \\
2004 & 0,040 & 25 & 0,003 \\
2005 & 0,038 & 26 & $-0,002$ \\
\hline
\end{tabular}

${ }^{a} \mathrm{El}$ inverso del IHH indica el número de empresas que se esperan en el mercado para un valor dado de $\mathrm{IHH}$.

mentar, los valores de este indicador se mantuvieron cercanos a cero, reflejo de una completa falta de elasticidad.

\section{DISCUSIÓN}

El mercado farmacéutico mexicano es un oligopolio en el que en 2005 un grupo reducido de 5 a 10 empresas controlaban 
entre $32,1 \%$ y $53,4 \%$ de un mercado inelástico en términos de precios. Es decir, como se ha mencionado en otros estudios, se trata de un mercado concentrado en el que los productores cuentan con un mayor poder relativo para fijar los precios que los consumidores (9).

De acuerdo con estudios previos, la concentración del mercado farmacéutico mexicano mostró una tendencia a una mayor concentración, si bien con algunas modificaciones en las empresas dominantes. Un estudio de 1996 encontró que en ese momento 10 empresas concentraban $33,0 \%$ del mercado (10).

Por su volumen de venta, el sector farmacéutico ocupa el segundo lugar en la economía manufacturera nacional, sólo por debajo de la industria petrolera. De acuerdo con los datos presentados, este sector ha incrementado sus ventas en $11,8 \%$ entre 2002 y 2005. Este crecimiento es mayor en $18,0 \%$ que el de la economía nacional (el producto interno bruto en ese mismo período aumentó $10,0 \%$ ) (7) y ratifica su ubicación como uno de los sectores más dinámicos de la economía mexicana.

Una particularidad del mercado farmacéutico mexicano es la clara diferenciación entre los sectores privado y público: si bien un porcentaje importante de las ventas se realiza directamente a los consumidores finales, los laboratorios que operan en México destinan esfuerzos considerables para constituirse en proveedores del sector público por el volumen que se puede negociar en un solo contrato. Esta estrategia, no obstante, es particularmente importante para los laboratorios nacionales (11).

En cuanto a su participación en el gasto total en salud, de acuerdo con las cifras del Sistema de Cuentas en Salud de la Secretaría de Salud de México, las ventas de productos farmacéuticos representaron $17,7 \%$ y $18,3 \%$ del total en los años 2002 y 2003, respectivamente (12).

La tendencia a mantener e incrementar el poder de mercado, concentrado en un grupo reducido de empresas, representa sin dudas un reto para su regulación, ya que esto crea incentivos para que esas empresas coludan y obtengan condiciones ventajosas para sus actividades comerciales.

Los posibles cambios en los patrones de compra -más concretamente en los porcentajes cubiertos por el sector público y los consumidores individuales- podrían modificar la estructura del mercado farmacéutico mexicano. Es importante con- tar con un panorama claro de la estructura del mercado y los grados de concentración existentes para analizar esos cambios y sus posibles consecuencias.

El marco regulatorio actual — que busca prevenir incrementos excesivos de los precios mediante límites vinculados con las variables macroeconómicas- no se ha respetado del todo, lo que ha dado lugar a propuestas que buscan relacionar los precios iniciales de los productos con los del mercado internacional, así como establecer ajustes asociados con la inflación (13). En otros países, las políticas de regulación de precios a partir de incentivos a la competencia con genéricos han mostrado resultados positivos (14). No obstante, esas políticas regulatorias podrían estar enfocándose demasiado en los aspectos industriales de la regulación y se estaría descuidando el papel fundamental de este mercado en la salud de la población (15).

En México, el poder relativo de los productores está generando precios socialmente ineficientes, que generan una demanda no óptima de los productos farmacéuticos. Al tratarse de bienes relacionados con la salud, esto podría estar teniendo consecuencias negativas en la salud de la población y el bienestar social. Se debe recordar que los precios elevados pueden ocasionar que los medicamentos no sean accesibles, especialmente para la población sin seguro de salud que debe pagarlos directamente de su bolsillo.

Aunque en México podría esperarse que las reformas orientadas a aumentar el porcentaje de las compras de medicamentos que se realizan a través de agencias como el Seguro Popular (un seguro de salud con contribución gubernamental y subsidios según el ingreso de la familia), se debería modificar la correlación de fuerzas en la fijación de los precios, ya que existen condiciones que podrían generar el efecto contrario. Por ejemplo, la descentralización del sector de la salud mexicano - es decir, el traslado a los estados de los recursos y la capacidad de decisión - ha propiciado que los gobiernos estatales negocien la compra de medicamentos de manera independiente, lo que reduce el poder que generaría hacer una compra consolidada nacional.

En un contexto en el que las principales necesidades de salud se concentran en la población de menores recursos, el acceso oportuno a los medicamentos básicos se convierte en una prioridad política. Los medicamentos son útiles únicamente si se encuentran al alcance de los que los necesitan; este es un elemento esencial que debe tomar en cuenta el marco regulatorio del mercado farmacéutico (16).

Desde el punto de vista de las herramientas de que disponen los ministerios de salud, un mecanismo factible para modificar la correlación de fuerzas son las negociaciones consolidadas para la compra de medicamentos, si bien esta estrategia podría ser funcional únicamente para el mercado controlado, en el que se concentran los medicamentos prescritos por los servicios públicos. Un ejemplo del poder de mercado que puede ejercer un monopsonio (una entidad que concentra todas las compras de un producto) son las compras consolidadas del Instituto Mexicano del Seguro Social. Esta experiencia puede expandirse para concentrar las negociaciones de compra de medicamentos con recursos públicos, de forma tal que sea posible negociar precios consistentes y mejores, acorde con la capacidad de pago del país. En teoría, las compras consolidadas podrían hacerse entre varios países para grupos de medicamentos, como se ha hecho ya para los antirretrovirales (17).

Por otra parte, una mayor competencia podría ser beneficiosa para los consumidores, como se ha observado en otros países. Sin embargo, no está claro hasta qué punto es factible la entrada de nuevas empresas en el mercado mexicano, especialmente si se toma en cuenta la importancia y el elevado costo de la investigación y el desarrollo en este sector (18). Esto hace que participen solo grandes empresas nacionales y extranjeras con capacidad de invertir en nuevas tecnologías y aplicaciones.

Se deben tomar en cuenta las experiencias de otros países de ingresos medios en la producción de medicamentos genéricos, especialmente cuando las condiciones de salud demandan una cantidad masiva de recursos. Es necesario considerar los elementos a favor y en contra, ya que algunas soluciones podrían reducir los incentivos a la investigación y el desarrollo en salud, en particular los relacionados con problemas específicos de los países en desarrollo $(19,20)$.

Los datos presentados en este análisis tienen algunas limitaciones. La más importante es que los cálculos de elasticidad se hicieron a partir de un producto índice y no de cada producto individual 
de la industria farmacéutica. Si bien esto limita el detalle del análisis, no hay razones para argumentar que esto modifica el resultado central del análisis.

Este trabajo constituye una primera caracterización del mercado farmacéu- tico mexicano, uno de los sectores más dinámicos de la economía nacional. Se comprobó que se trata de un mercado oligopólico e inelástico, por lo que se justifica la creación de mecanismos regulatorios más sólidos que reduzcan el poder

\section{REFERENCIAS}

1. KPMG Cárdenas Dosal. La industria farmacéutica en México. México, D.F.: KPMG; 2006. Hallado en http:/ /www.kpmg.com.mx/ publicaciones/libreria/mexico/st-farma ceutico(06).pdf. Acceso el 26 de junio de 2009.

2. Suárez G. México es el principal mercado farmacéutico de América Latina. Periódico Reforma 2005 febrero 7; Negocios: 2.

3. Economía/Empresas. El mercado farmacéutico mundial crecerá entre un $4,5 \%$ y un $5,5 \%$ en 2009, según IMS Health. Eleconomista. es [publicación en línea]. 2008 noviembre 3. Hallado en http://www.eleconomista.es/ economia/noticias/839439/11/08/EconomiaEmpresas-El-mercado-farmaceutico-mundialcrecera-entre-un-45-y-un-55-en-2009-segunIMS-Health.html. Acceso el 18 de julio de 2009.

4. Dávila E, Guijarro M. Evolución y reforma del sistema de salud en México. Santiago: Naciones Unidas; 2000.

5. Varian H. Microeconomía intermedia, un enfoque moderno. Barcelona: Antoni Bosch; 1994.

6. Instituto Nacional de Estadística, Geografía e Informática. Censos Económicos 2004. Industrias manufactureras. México: INEGI; 2006.

7. Banco de México. Informe Anual 2007. México, D.F.: Banxico; 2008.

8. Martin S. Advanced Industrial economics. Cambridge: Blackwell Publishers; 1993.
9. Guerra-Contreras R, Sáenz-Benítez I. Receta de mercado. Revista Ejecutivos de Finanzas 2006 febrero $1 ; 20-5$.

10. Rivas-Vilchis JF, Molina-Salazar R. Las especialidades farmacéuticas en México: análisis del mercado privado en 1996. Tem Selec Invest Clín. 1997;III(1):185-97.

11. Estados Unidos Mexicanos, Secretaría de Salud. Hacia una política farmacéutica integral para México. México, D.F.: Secretaría de Salud; 2005

12. Estados Unidos Mexicanos, Secretaría de Salud. Sistema de cuentas en salud a nivel federal y estatal. Bol Inf Estad. 2003;IV:23.

13. Organización para la Cooperación y el Desarrollo Económicos. Estudios de la OCDE sobre los sistemas de salud. México, D.F.: OCDE; 2005.

14. Dalen DM, Strom S, Haabeth T. Price regulation and generic competition in the pharmaceutical market. Eur J Health Econ. 2006;7(3): 204-11.

15. González-López-Valcárcel B, Pinilla-Domínguez J. Regulación, competencia y precios en los mercados de productos sanitarios. Gac Sanit. 2006;20(Supl2):3-13.

16. Pecoul B, Chirac P, Trouiller P, Pinel J. Access to essential drugs in poor countries: a lost battle? J Am Med Assoc. 1999;281(4):361-7. de los productores en favor de los consumidores. Entender el funcionamiento del mercado farmacéutico es un primer paso para desarrollar estrategias que incentiven su eficiencia en términos de su rendimiento social.
17. Organización Panamericana de la Salud. II Negociación Conjunta de Precios para Medicamentos Antirretrovirales en los Países de Sudamérica. Proceso y resultados. Washington, D.C.: OPS; 2006. Hallado en http:// www.paho.org/spanish/ad/fch/ai/II_Rond a_de_Negociacion_Procesos_Resultados.pdf. Acceso el 19 de julio de 2009.

18. Borrell JR, Merino-Castello A. Efectos perversos de la regulación farmacéutica en España: ¿hasta dónde se traslada la competencia? Gac Sanit. 2006;20(Supl2):41-50.

19. Barton JH. TRIPS and the global pharmaceutical market. Health Aff. 2004;23(3):146-54.

20. Bermúdez JAZ, Oliveira MA, de Oliveira EA. Expanding access to essential medicines in Brazil: recent regulation and public policies. In: Bermúdez JAZ, Oliveira MA, eds. Intellectual property in the context of the WTO TRIPS agreement: challenges for public health. Rio de Janeiro: ENSP/WHO, Oswaldo Cruz Foundation; 2004. Pp. 129-50.

Manuscrito recibido el 3 de diciembre de 2007. Aceptado para publicación, tras revisión, el 26 de julio de 2008.
ABSTRACT

\section{The pharmaceutical market in Mexico: size, value, and concentration}

Key words
Objectives. To describe the pharmaceutical drug market in Mexico in terms of its size, structure, business' market power, and consumer negotiating power.

Methods. A descriptive study based on data from the 2004 Economics Census and the reports of IMS Health, Inc. (Norwalk, Connecticut, United States of America). Sales amounts and volumes of Mexico's pharmaceutical companies from 2002-2005 were obtained and the Herfindahl-Hirschman Index (HHI) and its inverse were calculated as indicators of the market's degree of concentration; also, price elasticity was determined by a product index.

Results. The total value of the products manufactured by the pharmaceutical sector was 115 billion in 2006 Mexican pesos, of which 99\% pertained to companies categorized as large. This amount constituted $1.2 \%$ of the national gross domestic product that year $(20.0 \%$ of the health sector's portion, estimated to be $6.0 \%)$ and $3.9 \%$ of the total value of manufactured goods. The HHI of Mexico's pharmaceutical market during the study period was about 0.04 , albeit with a steady decline, and its inverse decreased from 23 to 26 . The price elasticity of pharmaceutical products was minimal (0.007, 0.003, and -0.002).

Conclusions. This study constitutes a preliminary description of Mexico's pharmaceutical market, one of the country's most dynamic economic sectors. It confirmed that the market is a rigid oligopoly, and thus supports enactment of firmer regulatory tools to reduce the power of the manufacturers in favor of that of the consumers. 